\title{
Crisis Communication Tactics of Chinese Internet Influencers
}

\author{
Shang Junyi \\ International Department of Cambridge, Tianjin Yinghua International School, Wuqing, Tianjin, China \\ Email: joyce20050224@gmail.com
}

\begin{abstract}
This essay aims to find out what crisis that internet influencers may possibly meet based on current examples and how they can act for effective crisis communications, some may be particular to Chinese internet influencers.

By collecting and analyzing the current examples of the crisis of Chinese internet influencers, I've summarized them into five types: improper remarks, messy private life, negative public influences, deceit, and management conflict.
\end{abstract}

Keywords: Internet influencers, crisis, public relations

\section{INTRODUCTION}

Nowadays, internet influencers are gradually seen as popular jobs, and the economy behind internet influencers is also seen as a trending market. The term 'internet influencer' is defined as 'people in real life or online who are followed by net users for an event or action then are known as influencers, or who export their expertise over the long term' [1]. Professional internet influencers who have already signed a contract with media companies usually make profits through these following methods: short video e-commerce, livestreaming e-commerce, fans' rewarding, advertisements, brand and product endorsements, and speculations. In this case, the public crisis will not only affect their fame and reputation but also affect their income and their company. I will summarize and discuss a few examples of the crisis of internet influencers below and sort them into different types in terms of causes, then compare and contrast the crisis communication methods they've used and what methods can be suggestive.

In order to clarify some of the key terms below, I want to introduce the image restoration theory suggested by William. L. Benoit in [2]. He stated that there are five types of possible image restoration methods, which are: denying, evasion of responsibility, reducing offensiveness of event, corrective action, and mortification. These key terms above will be further explained within the cases and link to the incident to help with understanding.

\section{CRISIS INTERNET INFLUENCERS MAY MEET AND CASE ANALYSIS}

In general, there are five major types of public crisis that internet influencers may meet.

\subsection{Improper remarks:}

The crisis of improper remarks is referred to as a public crisis in which someone particularly a public figure makes comments or remarks on things that are not allowed to comment on or that these comments are insulting or law-breaking. The crisis of improper remarks will cause severe impacts on the internet influencers from many aspects, sometimes they may even be asked for a penalty or detention.

There is a typical case of the crisis of improper remarks which is the incident of Chen Yifa, who is an internet anchor of both games and singing. In 2018 July, Chen Yifa was reported to the internet police and authoritative media for her insulting, sneering comments on the national painful memory such as Nanjing Massacre in her former live streaming in 2010, 2011, and 2016. Her unpatriotic and even nation-insulting remarks built up a negative and humiliating image and insulted her own nation, thus was condemned severely by the public. The next day the Douyu official published the decision of permanent an of Chen Yifa's account and then promised to set up specific regulations on management of the anchors' speech. Chen Yifa and her team reacted quickly right after the incident took place and published a letter of apology on Weibo, admitting her 
guilt. Her response is a typical example of mortification which is one of the five concepts in W. L. Benoit's image restoration theory [2], which is used aiming to lower the attitude and gain the public's forgiveness.

\subsection{Messy private life:}

The crisis of messy private life can be easily understood by its literal meaning, which is the exposure of the internet influencers' messy private life that seems to be immoral. The impact of messy private life being exposed is also quite serious, that it may severely worsen your public impression and cause condemnations, thus lowering popularization and the number of fans.

The incident of Banzangsenlin who is an internet influencer on Weibo can be used as an example of messy private life. She used to be exposed as the other woman in the relationship between Liu Yang and Qin in November 2019, and she has also criticized of her pictures posted on Weibo that contain little sexual innuendos, although it has already become her style of posts. After Qin has announced her break-up with Liu Yang and exposed Banzangsenlin's underground relationship with Liu Yang, the public soon started to question and condemn Banzangsenlin's behavior. She soon posted an apology statement explaining her relationship with Liu Yang as a mistake that she was just deluded by Liu Yang and thus forgot about the immorality. Mortification as an effective image restoration strategy [2] is used here in order to lower her position for more sympathy and prove that she has realized her fault. Although the public's response wasn't quite positive because she was shirking her responsibility, this apology statement did help her regain some reputation, and people that dislike her have no reason to condemn her further.

However, the other protagonist in this incident was treated harshly compared with Banzangsenlin. Like the other two ladies in this incident, Liu Yang is also an internet influencer on Weibo. His two-timer behavior immensely infuriated the public, and thus he has been blamed even more Banzangsenlin has. Furthermore, he was then exposed to be sexually harassing his female fans and female staff in his company during his relationship with Qin. This exposure with strong proofs of pictures and chatting records instantly causes the public particularly feminists' attention due to the suspicion of insulting female, very soon the public all started to concentrate their anger and censure on Liu Yang, which therefore indirectly reduces the blame on Banzangsenlin since she can be seen as a victim of Liu Yang, the lave rat. Liu Yang soon posted an apology letter as well, admitting that he was wrong and said that he did not expect anyone to forgive him. However, he refused to recognize those evident chatting records and pictures are true and said "I know I shouldn't.......anyway I've already done that...... I was totally wrong, I expect no comprehension' [4], which aroused the public's dissatisfaction that he did not directly apologize to his exgirlfriend Qin and he did not even regret. Liu Yang's mortification did not gain an overall positive public response as well, since he was just toughly evading responsibility and refused to talk about his fault just wanting to turn over the matter.

\subsection{Negative social influence}

Internet influencers as public figures have to be a positive model, at least need to rectify their own behaviors and don't call for others to do something bad. In this case, the crisis of negative social influence can be referred to that the internet influencer is a negative example instead of a positive model since his or her behavior is not suggested or promoted.

For instance, the incident of the internet influencer Wen Wan can be discussed as a typical example of having negative social influences. In 2018 when she became famous as an internet influencer by her short videos recorded in the underground parking garage, she was revealed to be only 17-year-old. Whereas based on little evidence from her social media, she was rumored that she went to nightclubs frequently, dropped out early from school, and leaned on rich people for money. As soon as these rumors have been revealed, Wen Wan appeared in the top search for days, and her ID was soon banned by the platform, she was abused as being a 'bad girl' thus her reputation soon fell to the bottom. However, because she had grabbed the public attention, she soon sighed a contract with a KOL company, becoming a professional internet influencer.

To whitewash Wen Wan's image, she was interviewed by a media contacted by her company after a few months, and the recorded video was soon posted on the internet [5]. In the video, she explained that these are only rumors, and she was just betrayed by one of her friends who secretly started this rumor with a media for unknown reasons. Furthermore, she expounded that she has a broken family and that she did drop out early from school but that was agreed by her mother, and her mother quite supported her to be an internet influencer since Wen Wan enjoyed it, although this job was actually quite exhausting. As soon as the video was posted, the public's rage and hatred lessened, because Wen Wan has successfully gained the public sympathy and approval that she appeared as an ordinary girl who is optimistic, moderate, and positive, and in response to the cyberviolence she has received during that period of time, she said that she has become stronger. The rumor seemed to be collapsed of itself, Wen Wan soon received a positive public response and her image was restored quickly in high proportion. This image restoration tactic Wen Wan has used is the combination of denial, evasion of responsibility, and reducing offensiveness of event, which successfully aroused the public sympathy and 
comprehension, and consequently, as some sort of a victim of the cyber-violence, accompanied with the denial of those negative rumors, Wen Wan's popularity was smoothly recovered and her fans came back one after another. Those negative, unreal exposures now are gradually forgotten by people and this could be seen as a case of successful image restoration of an internet influencer.

\subsection{Deceit}

The crisis of deceit appears quite common on Chinese internet influencers due to the advanced beauty effect in camera apps and developed PS technology. Many internet influencers are revealed to be not as pretty and handsome as they shown in pictures or videos, thus the crisis of deceit could involve.

A representative sample of internet influencers involves critically negatively-impactive deceit can be the incident of Qiao Biluo [3]. She used to be an anchor on Douyu, livestreaming games, and singing using an adorable voice. She always posted pictures of a beautiful girl and claimed that she will not reveal her real appearance during livestreaming until she has got one hundred thousand fans. Once in 2019, during her rewarding competition with another anchor, the picture which was used to hide her face disappeared due to bugs of the platform, and her real face was revealed to the audience ------ old, stocky, and homely, which looked different from the pictures in her social media. This tremendous psychological discrepancy caused many of her fans left, and the top one on her rewarding rank who had rewarded her one hundred thousand even deleted his account. This livestreaming accident quickly climbed up the top search and was soon known by the public. Qiao Biluo received overwhelming sarcasm and condemning due to her deceit and bragging, and even soon after she was found using another internet influencer's images as hers without a permit, which were the pictures she has posted on her social media. The next day, Douyu official announced that her livestreaming room has been closed permanently.

However, in order to make her popular again, Qiao Biluo posted an image of herself after make-up to prove that she was not that ugly as shown in the livestreaming and as what people commented, and asserted that she had had depression for 10 years. Furthermore, she attacked and blamed the anchor who was competing with her as being purposeful and vicious that she had planned all these to decrease her popularity. Her behaviors of tough, untrustworthy denial and foolish, unreasonable, proofless evasion of responsibility and accusation of the other anchor made the public even more outraged, resulting in a further deterioration in her reputation and public impression in an instant. Her livestreaming accident has even become a joke, and her incident was then called 'the transformation from a girl to a grandma'. In general, her response to the crisis is visibly a definite failure, which is not wisely planned and correctly fitting, that consequently, she became further infamous instead.

\subsection{Management conflict}

As stated in my introduction, nearly all popular internet influencers in China have signed contracts with either media companies, MCN companies, or KOL companies, and are manages centralized. Hence there might inevitably be communication and management conflicts between the internet influencer and the company, and this can be categorized as the crisis of management conflict.

One typical example of management conflict is the incident of Pan Nanqui. Pan Nanqui is a Korean lady who came to China years ago and became a professional internet influencer in Huoni Culture because of her beauty. On 25th April 2019, she posted a declaration complaining about the continuous violence she has been received from her superior, with pictures of her injuries. The commenting area was soon fulfilled with blames and questioning at Huoni Culture. Nonetheless, Huoni Culture's official account soon sent a formal announcement denying Pan Nanqui's accusation saying that it is Pan Nanqui whose temper was bad, whose mental state was bad and unstable, and who started the conflict first. The public opinion quickly swung to Huoni Culture and Pan Nanqui was then questioned. Furthermore, some media exposed that she has done numerous face-liftings spending one billion Korean Won that was rumored to be stolen from her ex-boyfriend. This scandal severely deteriorated Pan Nanqui's reputation and status, although her popularity did not decrease quite significantly ------ since she was a face-attractive internet influencer. However, Pan Nanqui did not make an active crisis communication response that she did not use any tactics of image restoration to repair her reduced reputation.

\section{SUGGESTIVE PUBLIC RELATIONS ACTIONS}

Although all the existing cases have comprised some public relations actions, some of them are not quite effective as expected. By analyzing all relevant cases, I want to introduce some suggestive public relations methods that are based on the image restoration theory. Priority among priorities, when facing severe public crisis like introduced above, people need to adjust their attitudes. Then depends on the types of crisis met, several strategies can be applied.

\subsection{Improper remarks:}

Take the incident of Chen Yifa as an example. First of all, because the problem is made by herself, active public apology is definitely essential, which shows the 
public that she have realized her fault. At the same time, saying it is only an accident, that's unintentional, promoting positive statements and other actions of reducing offensiveness can be applied as well. Then depends on the extent of severity, the influencer will probably need to pay some compensation like which Chen Yifa has, whether materially or based on the law. However, these strategies are not quite effective, since the types of improper remarks are variable, and that some might be unforgivable ------- like insulting countries, prejudice on sensitive social groups and so on.

\subsection{Messy private life:}

Virtually, this type of crisis normally will not have too much negative effects on popularity, unless the event has crossed the moral line or is illegal. However, for example in the incident of Banzangsenlin, it is quite crucial to show that the side with active immoral behavior, which includes herself and Liu Yang, has regretted and will make apology. Furthermore, both or all sides of the involvers can evade responsibilities by explaining that their actions are not intentional, or that it is inevitable. Or they can promise to do corrective actions or go against other involvers in order to reduce the public's offensiveness.

\subsection{Negative social influence:}

As exampled above in 2.3, Wen Wan's image restoration actions are quite effective, which it to wait until the heat of the event is much lower and then post vindicating herself and gaining sympathy by pouting out her hardness and conveying herself as a victim of the cyber-violence. Other resolutions for image restoration can be reducing offensiveness such as promoting positive things done before, making corrective actions and evading of responsibilities.

\subsection{Deceit}

The incident of Qiao Biluo's fake images is a typical negative example of response for image restoration. She has used denying trying to save her reputation, however, the fact that she has deceived and embezzled has been proved, thus her denying is not effective at all. In contrast, the most urgent action she should have done is to lower her attitude and make mortification. Furthermore, she can reduce people's offensiveness by promoting her virtue and specialty like her voice, to try to alter her way of attracting audiences, thus to remove the public's attention on her deception. Another choice is to depress the popularity of the event and fade out of the public's sight for a while, thus when this event is forgotten, the public rage will be reduced.

\subsection{Management conflict}

In case of the quarrel between Pan Nanqui and her superiors and colleagues, a quite effective means could be denying. Use pictures and posts showing the friendly relationships between her and the company and claiming that the misunderstanding has already been restored can transitorily reduce the public's anger, but evidences refuting those negative rumors of Pan Nanqui are more crucial. Besides, she can remove the public attention by publicize other merits of her and thus reduces the offensiveness.

\section{CONCLUSION}

Conclusively, the crisis communication tactics of five different sorts of crisis that Chinese internet influencers may meet are quite various. In comparison with the crisis communication tactics of firms and the government, I think the most significant difference is that the image restoration tactics Chinese influencers may use are all taken place on the Internet, with nearly no involvement of other kinds of media, and that is also one specific characteristic of the occupation. This characteristic could be considered as having many limitations that internet is a place where everyone concerns, hence every detail will be amplified that every mistake and negligence will be noticed, resulting in further negative butterfly effects. On the internet, influencers have to speak and act cautiously to protect themselves from being criticized and abused, thus prevent further crisis.

\section{REFERENCES}

[1] Baidu Baike, Influencer, n.d. [Online] Available from:https://baike.baidu.com/item/\%E7\%BD\%91 $\%$ Е7\%ВB\%9С\%Е7\%BA\%A2\%Е4\%BA\%BA/893 109 ?fromtitle $=\% \mathrm{E} 7 \% \mathrm{BD} \% 91 \% \mathrm{E} 7 \% \mathrm{BA} \% \mathrm{~A} 2 \&$ fro $\operatorname{mid}=6436044 \& \mathrm{fr}=$ aladdin

[2] William L. Benoit, Image restoration theory, 2015. DOI:https://doi.org/10.1002/9781405186407.wbiec i009.pub2

[3] Weibo, The whole story of Qiao BiluoDiscussing a mid-aged lady's tricky acting skills, 2019. [Online] Available from: https://weibo.com/ttarticle/p/show?id=2309404403 690864771119

[4] Douban, Banzangsenlin's shameless weibo, 2019 [Online] Available from: https://www.douban.com/group/topic/158540770/

[5] Weibo, Wen Wan claiming that she does not need white washing: she is who she is, 2020 [Online] Available

from: https://weibo.com/tv/show/1034:455449528021812 2?from=old_pc_videoshow 